\title{
MicroRNA-26a induces osteosarcoma cell growth and metastasis via the $\mathrm{Wnt} / \boldsymbol{\beta}$-catenin pathway
}

\author{
FENG QU ${ }^{1,2^{*}}$, CHUN-BAO LI ${ }^{2 *}$, BANG-TUO YUAN ${ }^{2}$, WEI QI ${ }^{2}$, HONG-LIANG LI ${ }^{2}$, \\ XUE-ZHEN SHEN ${ }^{2}$, GANG ZHAO ${ }^{2}$, JIANG-TAO WANG ${ }^{2}$ and YU-JIE LIU ${ }^{2}$ \\ ${ }^{1}$ Department of Orthopedics, Beijing Tongren Hospital, Capital Medical University, Beijing 100730; \\ ${ }^{2}$ Department of Orthopedics, Chinese People's Liberation Army General Hospital, Beijing 100853, P.R. China
}

Received February 27, 2015; Accepted December 4, 2015

DOI: $10.3892 / 01.2015 .4073$

\begin{abstract}
MicroRNAs (miRNAs/miRs) are a type of highly conserved, small non-coding RNA that are vital to the post-transcriptional regulation of gene expression via base pairing with target mRNA 3'-untranslated regions (3'-UTRs). Several studies have indicated that the abnormal expression of miRNAs occurs frequently in human osteosarcoma (OS). In the present study, the role of miR-26a in the progression and metastasis of OS was investigated using reverse transcription-quantitative polymerase chain reaction, a luciferase activity assay, cell viability assay, in vitro migration and invasion assays, transfection and western blot analysis. miR-26a was upregulated in OS tissues and cell lines, and the expression of miR-26a was indicated to affect the proliferation, migration and invasion of OS Saos- 2 cells. At the molecular level, the

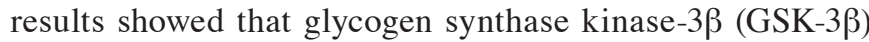
was identified as a target of miR-26a, and the ectopic expression of miR-26a inhibited GSK-3 $\beta$ by directly binding to the 3'-UTR. Therefore, the expression of miR-26a was negatively correlated with GSK-3 $\beta$ in the OS tissues. These data suggest that miR-26a is significant in the proliferation of human OS cells due to the direct regulation of $\mathrm{Wnt} / \beta$-catenin signaling.
\end{abstract}

\section{Introduction}

Osteosarcoma (OS) is the most common primary malignant bone tumor, occurring frequently in adolescents and possessing a high malignant severity (1-4). OS is commonly identified on the distal femur and proximal tibia, possessing high rates of recurrence and metastasis, and a poor prognosis. Previously, surgical resection therapy resulted in a poor prognosis for OS patients (2). At

Correspondence to: Professor Yu-Jie Liu, Department of Orthopedics, Chinese People's Liberation Army General Hospital, 28 Fuxing Road, Haidian, Beijing 100853, P.R. China

E-mail: liuandrew2014@hotmail.com

*Contributed equally

Key words: osteosarcoma, microRNA-26a, cell proliferation, glycogen synthase kinase- $3 \beta$, microRNAs present, the molecular pathogenesis and etiology of OS remain unclear. Therefore, the identification of the effector molecules or signaling pathways responsible for regulating tumor growth and metastasis is critical for improving OS treatment.

MicroRNAs (miRNAs/miRs) are a type of highly conserved, small non-coding RNA that are vital to the post-transcriptional regulation of gene expression via base pairing with target mRNA 3'-untranslated regions (3'-UTRs) (5,6). Previous studies have indicated that the abnormal expression of miRNAs is closely associated with cell proliferation, apoptosis, metastasis and invasion in human cancers, including OS $(7,8)$. miRNAs function as either tumor suppressors or oncogenes, depending on the role of the target genes. Previous studies have indicated that the inhibition of miR-26a may induce increased apoptosis in primary cultured chronic lymphocytic leukemia cells through suppression of phosphatase and tensin homolog (9). In addition, miR-26a inhibits hepatitis B virus transcription and replication by targeting the host factor cysteine and histidine-rich domain-containing, zinc-binding protein 1 (10).

In the present study, the miRNA expression profiles of human OS samples and cell lines were compared with those of adjacent normal skeletal muscle and normal cell lines. miR-26a was indicated to be upregulated in human OS and cell lines, and the expression of miR-26a affected the proliferation, migration and invasion of Saos- 2 cells subsequent to transfection with a miR-26a inhibitor or mimic. Additionally, miR-26a regulated glycogen synthase kinase-3 $\beta$ (GSK-3 $\beta$ ) expression through binding to the 3'-UTR of GSK-3 $\beta$ mRNA. miR-26a expression was negatively correlated with GSK-3 $\beta$ expression in OS tissues.

\section{Materials and methods}

Cell culture and tissue samples. In total, 20 paired OS and matched normal non-tumor tissues were obtained during biopsy from patients at the Department of Orthopedics, Chinese People's Liberation Army (PLA) General Hospital (Beijing, China). All tissues were immediately stored in liquid nitrogen until use. The present study was approved by the Ethics Committee of the Chinese PLA General Hospital.

Human OS U2OS, Saos-2, HOS and MG-63 cell lines were obtained from the American Type Culture Collection (Manassas, VA, USA) and cultured in Dulbecco's modified Eagle's medium 
(DMEM; Hyclone, Beijing, China) and Roswell Park Memorial Institute (RPMI)-1640 medium (Hyclone), supplemented with $10 \%$ fetal bovine serum (FBS; Hyclone), $100 \mathrm{mg} / \mathrm{ml}$ streptomycin (Hyclone) and $100 \mathrm{IU} / \mathrm{ml}$ penicillin (Hyclone) at $37^{\circ} \mathrm{C}$, in $5 \%$ $\mathrm{CO}_{2}$. The human osteoblast hFOB 1.19 cell line (American Type Culture Collection) was maintained in DMEM/F12 medium (Hyclone) supplemented with $10 \%$ FBS, $100 \mathrm{mg} / \mathrm{ml}$ streptomycin and $100 \mathrm{IU} / \mathrm{ml}$ penicillin at $37^{\circ} \mathrm{C}$, in $5 \% \mathrm{CO}_{2}$.

RNA isolation and reverse transcription-quantitative polymerase chain reaction $(R T-q P C R)$. Total RNA and $\mathrm{miR}$ were isolated using the RNeasy Mini and miRNeasy Mini kits (Qiagen, Inc., Valencia, CA, USA), according to the manufacturer's protocol. For the miRNA expression assay, reverse transcription and RT-qPCR were performed using Applied Biosystems TaqMan miRNA assay kits (Thermo Fisher Scientific, Waltham, MA, USA) and Applied Biosystems hsa-miR-26a (cat. no. 4395166; Thermo Fisher Scientific). PCR reactions were performed using a Bio-Rad iCycler iQ RealTime PCR Detection System (Bio-Rad Laboratories, Hercules, CA, USA), 20 ng cDNA and the following primers (Sangon Biotech Co., Ltd., Shanghai, China): Forward, 5'-TTGGATCCGTCAGAAATTCTCTCCCGAGG-3' and reverse, 5'-GGTCTAGATGTGAACTCTGGTGTTGGTGC-3' for miR-26a; forward, 5'-GGAGAACTGGTCGCCATCAAG-3' and reverse, 5'-ACATTGGGTTCTCCTCGGACC-3' for GSK-3 $\beta$; and forward, 5'-GCGCGTCGTGAAGCGTTC-3' and reverse, 5'-GTGCAGGGTCCGAGGT-3' for U6. PCR was performed under the following conditions: Denaturation, $95^{\circ} \mathrm{C}$ for $10 \mathrm{~min}$, followed by 35 cycles of $95^{\circ} \mathrm{C}$ for $15 \mathrm{sec}$ and $60^{\circ} \mathrm{C}$ for $1 \mathrm{~min}$, and melting curve analysis at $95^{\circ} \mathrm{C}$ for $15 \mathrm{sec}, 60^{\circ} \mathrm{C}$ for $1 \mathrm{~min}, 95^{\circ} \mathrm{C}$ for $15 \mathrm{sec}$ and $60^{\circ} \mathrm{C}$ for $15 \mathrm{sec}$. The RT-qPCR data were normalized using the $2^{-\Delta \Delta \mathrm{Cq}}$ method (11), relative to glyceraldehyde-3-phosphate dehydrogenase or U6 small nuclear RNA.

miRNA transfection. Transfections of miRNA were performed using Invitrogen Lipofectamine 3000 (Thermo Fisher Scientific), according to the manufacturer's protocols. miR-26a mimic and control mimics (Ribobio Co., Ltd., Guangzhou, China) were used at a final concentration of $100 \mathrm{nM}$. For the miR-26a inhibitor, an inhibitor control (Ribobio Co., Ltd.) was added to the transfection complexes at a final concentration of $20 \mathrm{nM}$. The medium was changed following 4-6 h incubation in a $\mathrm{CO}_{2}$ incubator at $37^{\circ} \mathrm{C}$.

Luciferase activity assay. A luciferase activity assay was performed, as previously described (12). Briefly, Saos-2 cells were cultured in a 12 -well plate $\left(1 \times 10^{5}\right.$ cells/well $)$, and co-transfected with wild-type (WT) or mutated (Mut) 3'-UTRs of GSK-3 $\beta$, luciferase reporter constructs and either miR-26a or a control using Invitrogen Lipofectamine 3000. The cells were harvested and the luciferase activity was examined $24 \mathrm{~h}$ later, using the Dual-Luciferase Reporter Assay kit (Promega Corporation, Madison, WI, USA).

Cell viability assay. The cell counting kit-8 (CCK-8; Dojindo Molecular Technologies, Inc., Kumamoto, Japan) assay was used as a qualitative index of cell viability, which was based on the conversion of a water-soluble tetrazolium salt, 2-(2-methoxy-4
-nitrophenyl)-3-(4-nitrophenyl)-5-(2,4-disulfophenyl)-2H-tetrazolium monosodium salt (WST-8), to a water soluble formazan dye upon reduction by dehydrogenases in the presence of an electron carrier (13). The cells were plated in 96-well microplates, then the cell count was obtained using the CCK-8 assay, according to the manufacturer's protocols. Briefly, $10 \mu \mathrm{l}$ of CCK-8 solution was added to each well, and the samples were incubated for $1 \mathrm{~h}$, prior to measuring the absorbance at $450 \mathrm{~nm}$.

Colony formation assay. In total, 500 transfected Saos-2 cells were seeded into a 6-well plate and maintained in DMEM containing 10\% FBS for 14 days. Next, the cells were fixed and stained with methanol for $20 \mathrm{~min}$, followed by $0.5 \%$ crystal violet for $15 \mathrm{~min}$. Visible colonies were quantified using an inverted microscope (IX83; Olympus Corporation, Tokyo, Japan).

In vitro migration and invasion assay. Migration and invasion assays were performed using Transwell chambers (BD Biosciences, Franklin Lakes, NJ, USA). For the migration assay, $5 \times 10^{4}$ cells were seeded into the upper chamber of the Transwell. For the invasion assay, $1 \times 10^{5}$ cells were added into the upper chamber of the Transwell, which was precoated with Matrigel (BD Biosciences). In each assay, the cells were maintained in DMEM without serum in the upper chamber, and DMEM containing 10\% FBS was added to the lower chamber to act as a chemoattractant. Following $24 \mathrm{~h}$ of incubation, the cells that did not migrate or invade through the membrane were removed. Next, the membranes were fixed and stained with $0.5 \%$ crystal violet. Four random fields were counted per chamber using an inverted microscope (IX53; Olympus Corporation), and each experiment was repeated 3 times.

Western blot analysis. The cells or tissues were harvested and lysed using ice-cold lysis buffer [50 mM Tris-HCl ( $\mathrm{pH} 6.8$ ), $32 \mathrm{mM}$ 2-mercaptoethanol, $2 \% \mathrm{w} / \mathrm{v}$ sodium dodecyl sulfate (SDS) and $10 \%$ glycerol]. Following centrifugation at $20,000 \mathrm{x} \mathrm{g}$ for $10 \mathrm{~min}$ at $4^{\circ} \mathrm{C}$, the proteins in the supernatants were quantified and separated using $10 \%$ SDS polyacrylamide gel electrophoresis and transferred to a nitrocellulose membrane (GE Healthcare Life Sciences, Chalfont, UK). Subsequent to blocking with $10 \%$ skimmed milk in phosphate-buffered saline, the membranes were immunoblotted with antibodies as indicated, followed by horseradish peroxidase-linked goat anti-rabbit IgG secondary antibodies (cat. no. ab6721; 1:10,000; Santa Cruz Biotechnology, Inc., Dallas, TX, USA). The signals were detected using the SuperSignal West Pico Chemiluminescent Substrate kit (Pierce Biotechnology, Inc., Rockford, IL, USA), according to the manufacturer's protocols. Monoclonal rabbit anti-human GSK-3 $\beta$ (cat. no. ab32391; 1:1000), $\beta$-catenin (cat. no. ab32572; 1:3,000), c-Myc (cat. no. ab32072; 1:1,000), Cyclin D1 (cat. no. ab134175; $1: 1,000)$ and $\beta$-actin (cat. no. ab6276; 1:10,000) antibodies were purchased from Abcam (Cambridge, MA, USA). Protein levels of $\beta$-actin were employed as loading controls. The relative expression level of proteins was analyzed by Quantity One software (Bio-Rad Laboratories).

Statistical analysis. Data are presented as the mean \pm standard deviation, and were analyzed using the analysis of variance or two-tail Student's t-test to examine the statistical significance. $\mathrm{P}<0.05$ was considered to indicate a statistically significant 
A

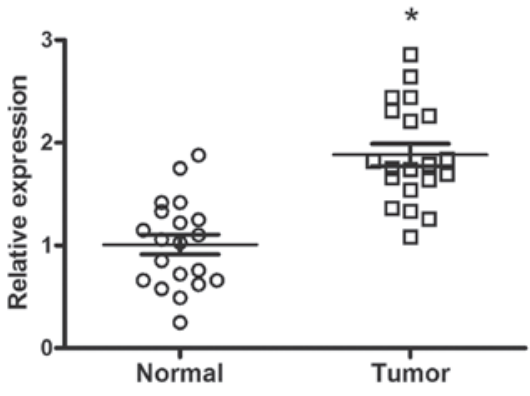

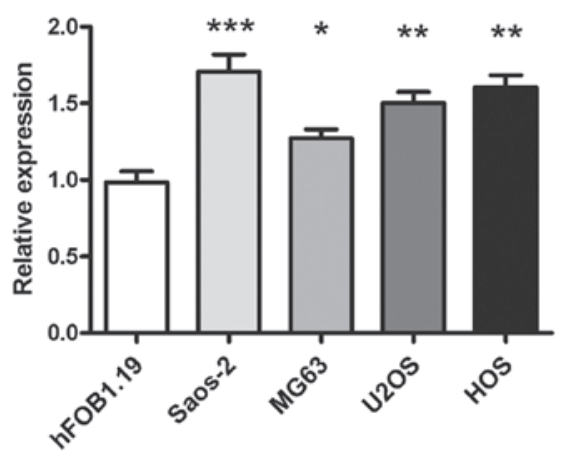

Figure 1. miR-26a expression is increased in OS tissues and cell lines. (A) miR-26a was significantly increased in the OS tissues compared with the corresponding normal tissues. (B) miR-26a was significantly increased in the OS U2OS, Saos-2, HOS and MG-63 cell lines, compared with the human osteoblast hFOB 1.19 cell line. ${ }^{*} \mathrm{P}<0.05,{ }^{* *} \mathrm{P}<0.01$ and ${ }^{* * *} \mathrm{P}<0.001$ compared with the control group. OS, osteosarcoma; miR-26a, microRNA-26a.

A

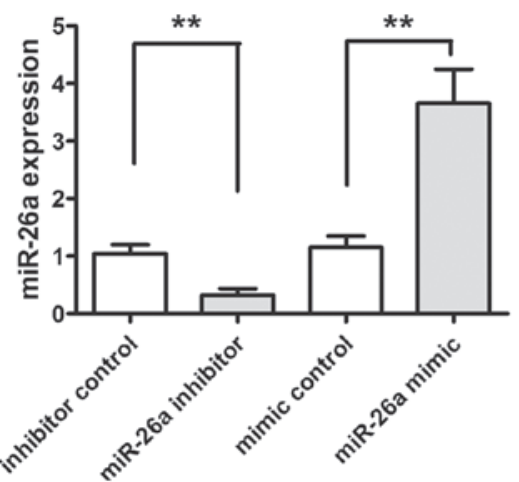

D

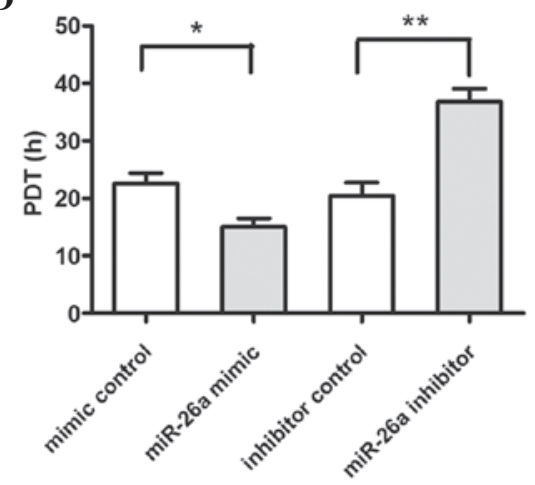

B

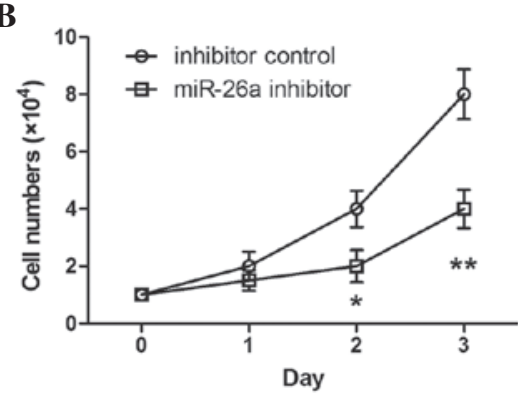

E

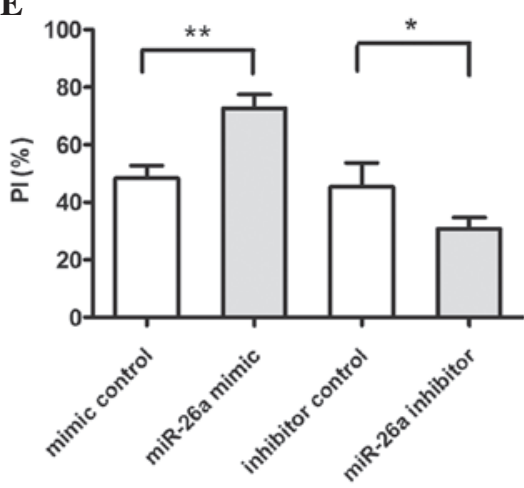

G

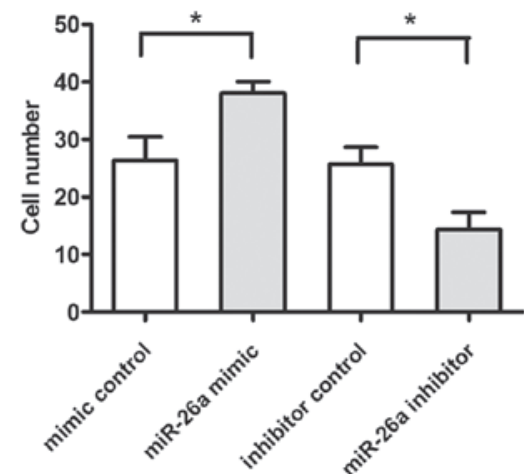

C

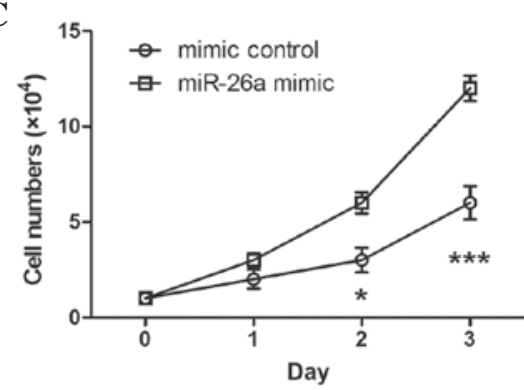

F

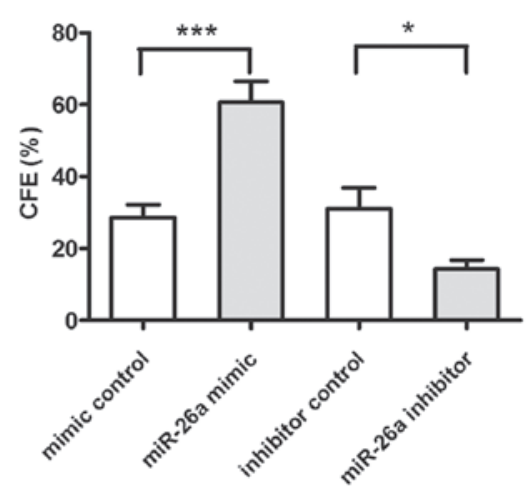

Figure 2. Various levels of miR-26a affect the proliferation, migration and invasion of osteosarcoma cells. (A) The expression of miR-26a was measured at $48 \mathrm{~h}$ post-transfection using reverse transcription-quantitative polymerase chain reaction. Cell viability of the (B) inhibitor and (C) mimic control was measured using a cell counting kit-8 assay. (D) Cell PDT was determined at $48 \mathrm{~h}$ post-transfection. (E) Cell cycle analysis was performed at $48 \mathrm{~h}$ post-transfection by staining DNA with propidium iodide prior to flow cytometry. (F) Clonogenic assay was performed at $24 \mathrm{~h}$ post-transfection. (G) Cells migrating across the membrane were counted in all fields. Data was analyzed using the Student's t-test and is expressed as the mean \pm standard deviation from three independent experiments. ${ }^{*} \mathrm{P}<0.05 ;{ }^{* *} \mathrm{P}<0.01 ;{ }^{* * *} \mathrm{P}<0.001$. PDT, population doubling time; PI, proliferative index; CFE, colony-forming efficiency; miR-26a, microRNA-26a. 
A
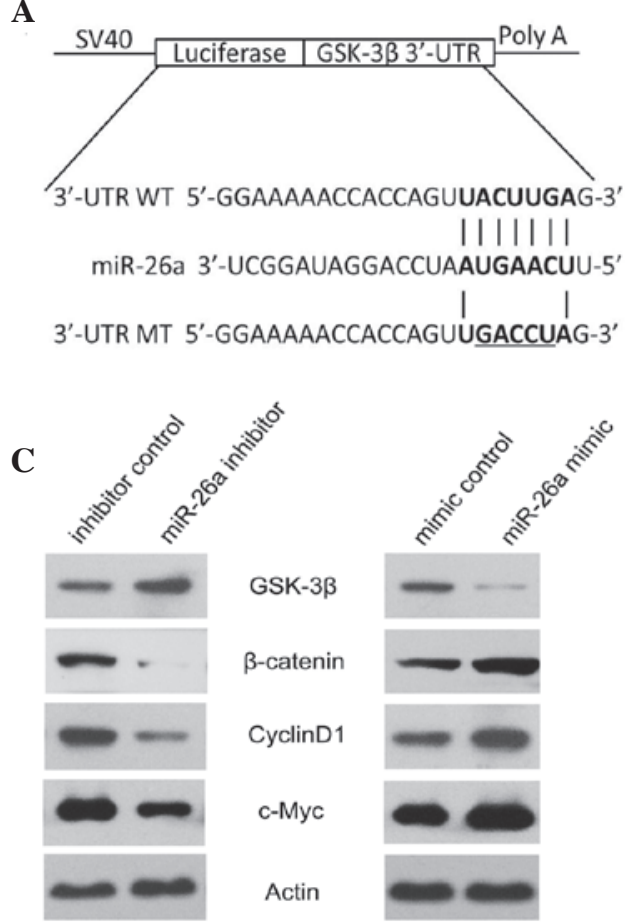



D

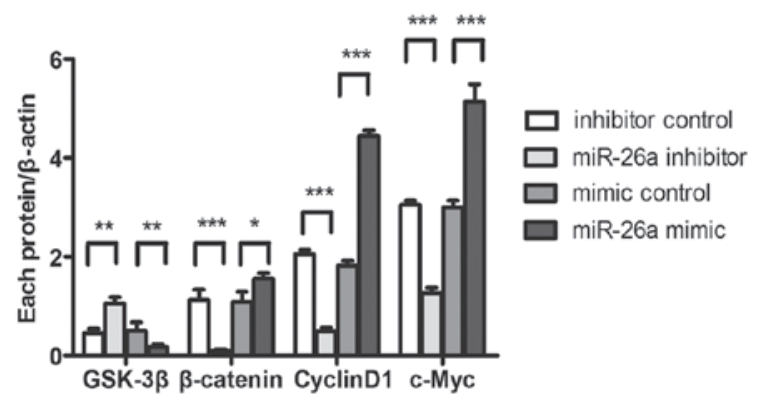

Figure 3. GSK-3 $\beta$ is a direct target of miR-26a. (A) Computational analysis showed that miR-26a potentially targeted GSK-3 $\beta$. (B) Saos-2 cells were co-transfected with miR-26a and WT or Mut 3'-UTR luciferase reporter constructs. (C) Protein levels of GSK-3 $\beta$, $\beta$-catenin, cyclin D1, c-Myc and $\beta$-actin were detected using western blot analysis in Saos-2 cells that were transfected with miR-26a inhibitor/inhibitor control or miR-26a mimic/mimic control. (D) Quantitative analysis was performed on each protein level using Quality One software. ${ }^{*} \mathrm{P}<0.05,{ }^{* *} \mathrm{P}<0.01$ and ${ }^{* * *} \mathrm{P}<0.001$ compared with the control group. GSK-3 $\beta$, glycogen synthase kinase-3 $\beta$; WT, wild-type, Mut, mutated; UTR, untranslated region; miR, microRNA; ctrl, control.

A

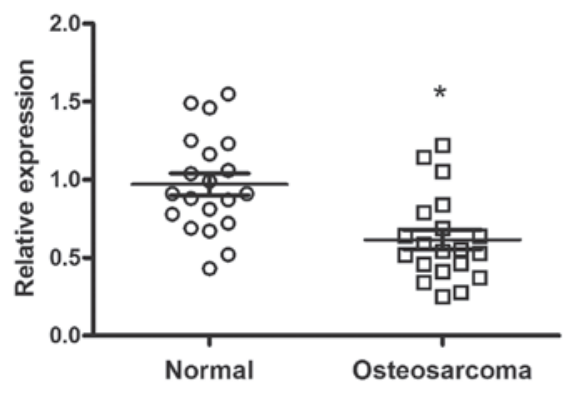

B

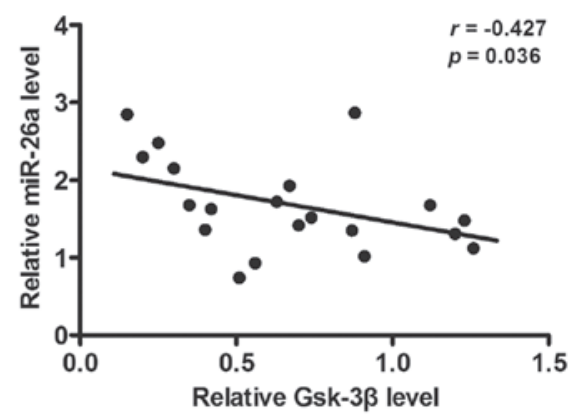

Figure 4. miR-26a expression is negatively correlated with GSK-3 $\beta$ expression in OS tissues. (A) GSK-3 $\beta$ mRNA level was examined using reverse transcription-quantiative polymerase chain reaction and was markedly decreased in the OS tissues. (B) GSK-3 $\beta$ mRNA level was inversely correlated with miR-26a level in the OS tissues (Spearman's rank correlation coefficient; $r=-0.427 ; \mathrm{P}=0.036$ ). ${ }^{*} \mathrm{P}<0.05$ compared with the control group. GSK-3 $\beta$, glycogen synthase kinase-3 $\beta$; OS, osteosarcoma; miR-26a, microRNA-26a.

difference. The correlation between GSK-3 $\beta$ and miR-26a expression was assessed using Spearman's rank correlation coefficient.

\section{Results}

miR-26a is upregulated in human OS tissues and cell lines. The expression of miR-26a in 20 OS tissues and matched normal tissues was determined by RT-qPCR. The expression of miR-26a was significantly increased in the OS tissues compared with the matched normal tissues ( $\mathrm{P}=0.027$; Fig. 1A). In addition, the expression of miR-26a in the OS U2OS $(\mathrm{P}=0.0093)$, Saos-2, HOS $(\mathrm{P}=0.0088)$ and MG-63 cell lines was markedly increased compared with the human osteoblasthFOB 1.19 cell line (Fig. 1B).
Expression of miR-26a affects the proliferation, migration and invasion of Saos-2 cells. To examine the role of miR-26a in OS cell growth, Saos-2 cells were transfected with miR-26a inhibitor/inhibitor control or miR-26a mimic/mimic control, and the results were detected by RT-qPCR (Fig. 2A). The RT-qPCR results indicated that transfection with the miR-26a inhibitor significantly reduced miR-26a expression $(\mathrm{P}=0.0075)$ and transfection with the miR-26a mimic significantly increased miR-26a expression $(\mathrm{P}=0.0084)$. The results of CCK8 assay indicated that the proliferation rate of the cells transfected with the miR-26a inhibitor was significantly decreased compared with the inhibitor control cells (Fig. 2B): $\mathrm{P}=0.048$ on day 2 and $\mathrm{P}=0.0089$ on day 3 . The transfected miR-26a mimic results 
indicated that the proliferation rate of the cells transfected with miR-26a mimic was significantly increased compared with the mimic control cells (Fig. 2C): $\mathrm{P}=0.038$ on day 2 and $\mathrm{P}=0.0008$ on day 3 . In addition, the cells transfected with the miR-26a inhibitor possessed a significantly longer population doubling time, decreased proliferative index and colony forming efficiency compared with control cells; furthermore, the cells transfected with the miR-26a mimic had a shorter population doubling time $(\mathrm{P}=0.0041)$, increased proliferative index $(\mathrm{P}=0.047)$ and colony forming efficiency $(\mathrm{P}=0.048)$ compared with the control group (Fig. 2D-F). Additionally, the number of cells migrating across the membrane, with or without Matrigel, that demonstrated miR-26a-knockdown (miR-26a inhibitor) was significantly decreased $(\mathrm{P}=0.038)$ compared with that in the control cells (Fig. 2G). These data provide strong evidence that the knockdown of miR-26a may inhibit proliferation, migration and invasion in Saos-2 cells, and that transfection with miR-26a may increase cell proliferation, migration and invasion.

miR-26a downregulates GSK-3 $\beta$ through binding to the

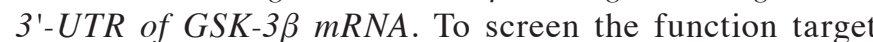
of miR-32 in OS cells, bioinformatics software (miRWalk; http://www.umm.uni-heidelberg.de/apps/zmf/mirwalk/) was used. The gene encoding GSK-3 $\beta$ was indicated to harbor a potential miR-26a binding site (Fig. 3A). Luciferase reporter assays using the 3 '-UTR of the GSK-3 $\beta$ gene demonstrated that the miR-26a mimic significantly decreased the activity of the GSK-3 $\beta$ 3'-UTR $(\mathrm{P}=0.0096)$, but not the binding motif mutant 3'-UTR (Fig. 3B). In addition, the overexpression of miR-26a significantly suppressed the GSK-3 $\beta$ protein level. The intracellular $\beta$-catenin protein level increased, as less $\beta$-catenin was degraded through GSK-3 $\beta$, which resulted in the activation of Wnt $/ \beta$-catenin signaling pathways. Downstream target genes of $\beta$-catenin, including Cyclin D1 and c-Myc, were activated, and the proteins stimulated the growth and metastasis of the tumor cells (Fig. 3C and D). The opposite result was indicated in the cells that were transfected with miR-26a inhibitor.

miR-26a expression is negatively correlated with GSK-3 $\beta$ expression in OS tissues. The expression of GSK-3 $\beta$ mRNA in 20 OS samples and the corresponding normal tissues was measured. Results indicated that the expression of GSK-3 $\beta$ mRNA was significantly decreased in OS tissues compared with the corresponding normal tissues ( $\mathrm{P}=0.044$; Fig. 4A). In addition, GSK-3 $\beta$ expression was inversely correlated with miR-26a levels in OS tissues ( $\mathrm{P}=0.036$; Fig. 4B).

\section{Discussion}

The elucidation of functional targets is one of the best ways to understand the function of miRNA; this typically involves an analysis of the changes in target proteins, following a gain or loss of function of the specific miRNA. The present results indicated that miR-26a expression was upregulated in the OS tissues and cell lines. Forced overexpression of miR-26a promoted cell proliferation, migration and invasion in Saos-2 cells, while miR-26a inhibition suppressed cell proliferation and metastasis. Additionally, GSK-3 $\beta$ was indicated to be a target of miR-26a, and miR-26a expression was negatively correlated with GSK-3 $\beta$ expression in the OS tissues. However, additional studies are required in order to investigate the role of miR-26a in vivo.

Abundant miR-26a expression is present in the heart tissues of rats and humans $(14,15)$, with decreased levels in ischemic preconditioning. Studies into the role of miR-26a have also been performed in a number of cancer cell types. In breast cancer cells, miR-26a has been demonstrated to initiate cell apoptosis through the extrinsic and intrinsic pathways by caspase- 8 and caspase-9 activation, respectively (16). In the nasopharyngeal carcinoma C666-1 cell line, apoptosis induced by ionizing radiation was dependent on reactive oxygen species, and exogenous miR-26a expression resulted in significant toxicity in the cells (17). However, the role of miR-26a in OS remains unclear.

Overall, the present study demonstrated an association between miR-26a and GSK-3 $\beta$, and provided a mechanism for OS cell growth, migration and invasion. These results suggest that miR-26a may act as an oncogene in OS and represent a potential molecular target for OS therapy.

\section{References}

1. Bao YP, Yi Y, Peng LL, et al: Roles of microRNA-206 in osteosarcoma pathogenesis and progression. Asian Pac J Cancer Prev 14: 3751-3755, 2013.

2. He ML, Wu Y, Zhao JM, Wang Z and Chen YB: PIK3CA and AKT gene polymorphisms in susceptibility to osteosarcoma in a Chinese population. Asian Pac J Cancer Prev 14: 5117-5122, 2013.

3. Jia J, Tian Q, Liu Y, Shao ZW and Yang SH: Interactive effect of bisphenol A (BPA) exposure with -22G/C polymorphism in LOX gene on the risk of osteosarcoma. Asian Pac J Cancer Prev 14: 3805-3808, 2013

4. Jiang W, Huang Y, Wang JP, Yu XY and Zhang LY: The synergistic anticancer effect of artesunate combined with allicin in osteosarcoma cell line in vitro and in vivo. Asian Pac J Cancer Prev 14: 4615-4619, 2013.

5. Ameres SL and Zamore PD: Diversifying microRNA sequence and function. Nat Rev Mol Cell Biol 14: 475-488, 2013.

6. Sun K and Lai EC: Adult-specific functions of animal microRNAs. Nat Rev Genet 14: 535-548, 2013.

7. Miao J, Wu S, Peng Z, Tania M and Zhang C: MicroRNAs in osteosarcoma: Diagnostic and therapeutic aspects. Tumour Biol 34: 2093-2098, 2013.

8. Nugent M: MicroRNA function and dysregulation in bone tumors: The evidence to date. Cancer Manag Res 6: 15-25, 2014.

9. Zou ZJ,Fan L, Wang L, et al: miR-26a and miR-214 down-regulate expression of the PTEN gene in chronic lymphocytic leukemia, but not PTEN mutation or promoter methylation. Oncotarget 6: 1276-1285, 2015

10. Zhao F, Xu G, Zhou Y, et al: MicroRNA-26b inhibits hepatitis $\mathrm{B}$ virus transcription and replication by targeting the host factor CHORDC1 protein. J Biol Chem 289: 35029-35041, 2014.

11. Livak KJ and Schmittgen TD: Analysis of relative gene expression data using real-time quantitative PCR and the 2(-Delta Delta C(T)) Method. Methods 25: 402-408, 2001.

12. $\mathrm{Li} \mathrm{H}$ and Yang BB: Stress response of glioblastoma cells mediated by miR-17-5p targeting PTEN and the passenger strand miR-17-3p targeting MDM2. Oncotarget 3: 1653-1668, 2012.

13. Han SB, Shin YJ, Hyon JY and Wee WR: Cytotoxicity of voriconazole on cultured human corneal endothelial cells. Antimicrob Agents Chemother 55: 4519-4523, 2011.

14. Dong S, Cheng Y, Yang J, et al: MicroRNA expression signature and the role of microRNA-21 in the early phase of acute myocardial infarction. J Biol Chem 284: 29514-29525, 2009.

15. Li Q, Song XW, Zou J, et al: Attenuation of microRNA-1 derepresses the cytoskeleton regulatory protein twinfilin-1 to provoke cardiac hypertrophy. J Cell Sci 123: 2444-2452, 2010.

16. Zhang B, Liu XX, He JR, et al: Pathologically decreased miR-26a antagonizes apoptosis and facilitates carcinogenesis by targeting MTDH and EZH2 in breast cancer. Carcinogenesis 32: 2-9, 2011.

17. Alajez NM, Shi W, Hui AB, et al: Enhancer of Zeste homolog 2 (EZH2) is overexpressed in recurrent nasopharyngeal carcinoma and is regulated by miR-26a, miR-101, and miR-98. Cell Death Dis 1: e85, 2010. 